\title{
Symmetric Atlasing and Model Based Segmentation: An Application to the Hippocampus in Older Adults
}

\author{
Günther Grabner ${ }^{1,2}$, Andrew L. Janke ${ }^{1}$, Marc M. Budge ${ }^{2}$, David Smith ${ }^{3}$, \\ Jens Pruessner ${ }^{1}$, and D. Louis Collins ${ }^{1}$ \\ ${ }^{1}$ McConnell Brain Imaging Centre, Montreal Neurological Institute, Quebec, Canada \\ ${ }^{2}$ The Australian National University, Canberra, Australia \\ ${ }^{3}$ Oxford University, UK
}

\begin{abstract}
In model-based segmentation, automated region identification is achieved via registration of novel data to a pre-determined model. The desired structure is typically generated via manual tracing within this model. When model-based segmentation is applied to human cortical data, problems arise if left-right comparisons are desired. The asymmetry of the human cortex requires that both left and right models of a structure be composed in order to effectively segment the desired structures. Paradoxically, defining a model in both hemi-spheres carries a likelihood of introducing bias to one of the structures. This paper describes a novel technique for creating a symmetric average model in which both hemispheres are equally represented and thus left-right comparison is possible. This work is an extension of that proposed by Guimond et al [1]. Hippocampal segmentation is used as a test-case in a cohort of 118 normal eld-erly subjects and results are compared with expert manual tracing.
\end{abstract}

\section{Introduction}

Non-subjective segmentation of sub-cortical structures from MRI has plagued the automated analysis of large clinical data sets. This is especially true when dealing with cohorts of patients exhibiting widely varying anatomical structure. Methods involving manual tracing suffer from inter- and intra-operator variability 2 and the increase in tracing effort required for high resolution data sets. Automated segmentation allows consistent analysis of structure volumes to be performed thus enabling non subjective estimates of atrophy in cohorts. Model based segmentation relies upon a pre-existing notion or manual delineation of the chosen structure on an average model of anatomy that represents the patient population being studied. The notion of an anatomical model is by no means new, digital atlases of both the human and animal cortices have been available for some time. Early instances of such models include a rat brain [3], a human model from CT data [4, and others [56. Many of these early models were derived from a single subject's data, and therefore could not represent the anatomical variability present in a population. Toga et al [7] provides a good summary of these recent developments. 
In order to build these models various linear and non-linear registration techniques have been developed to align individual subject data that constitutes the average. These registration techniques also allow labels that have been defined on models to be transformed onto individual patients anatomy by using the inverse of the subject-to-model transformation. Gee et al [8] proposed a system whereby $3 \mathrm{D}$ atlases of anatomy could be matched to anatomical images.

Both Thompson [9] and Davatzikos [10] proposed a more sophisticated method whereby the cortex of individuals could be modeled via a deformable model. Despite the differences in methodology, the eventual goal of all these methods is to produce an average model that can accurately both show the similarities in the group and exclude the effect of anatomic variance.

Wang and Joshi et al [1] expanded upon these methods in order to determine left-right differences of the hippocampus (HC). An inherent problem with the current approach to average modeling used in these methodologies to determine initial struc-ture size is the asymmetry in the cortex. As such, if a structure is outlined in both the left and right sides or left/right comparisons are required in a model, a bias will be introduced if the initial delineations on the model differ in shape or volume. This bias will be made apparent during the fitting of novel data to a model as the individual hemispheres will require differing parameters to achieve a robust fit. In a normal aver-age model of cortical structure this will be the case 12 .

Here an approach that annuls this effect without compromising the automatic seg-mentation performance is presented. In this methodology, images are iteratively matched linearly and non-linearly to an evolving model of average structure. This is similar to the procedure described by Guimond 1. At each stage a new model is built by averaging the current registration results. The technique presented here is different in that at each stage images are matched to the model in both their original and (Left-Right) mirrored orientation.

The aims of this methodology were to:

1. Build a high resolution symmetric atlas from a population.

2. Automatically extract sub-cortical structures in a population.

3. Allow left-right volumetric comparisons to be made without bias.

4. Eliminate subjective error.

\section{Methods}

For this work, 153 T1-volumetric brain MRI scans from the Oxford Project to Investi-gate Memory and Ageing (OPTIMA) were used. These data-sets were acquired on a $1.5 \mathrm{~T}$ System with a spatial resolution of $0.86 \mathrm{x} 0.86 \mathrm{x} 2 \mathrm{~mm}$.

\subsection{Data Pre-processing}

The data was converted to the MINC format and intensity corrected using histogram spline sharpening [13. Image intensity was then clamped between the 0.1 st and 99.9th percentile and this range of values is rescaled between 0 and 
100. In this case the chosen range is arbitrary. Clamping is performed to reduce image artifacts (typically high-signal background noise) and bring images into approximate intensity alignment. After the first generation model was created, the individual volume intensities were normalized against the model based on the median intensity values [17.

\section{$2.2 \quad$ Registration Methods}

The linear and non-linear registration methods used in this paper are slight modifica-tions of the non-linear matching strategy (mritotal + ANIMAL) proposed by Collins et al [14, the only difference being in the number of iterations used during each stage of non-linear fitting (as shown in Table 1). As proposed by 14, the ANIMAL algo-rithm comprises a hierarchical approach to registration. An initial linear fit is per-formed followed by progressively higher-resolution nonlinear fits. This procedure results in an affine transformation with an associated vector-volume to describe the non-linear component of the fit. The objective function used in the fitting is primarily cross-correlation and the regularization model is linear-elastic.

\subsection{Symmetric Model Generation}

In iteration 0 each aligned subject data-set is linearly registered to the initial model (ICBM152 - symmetric [packages.bic.mni.mcgill.ca]) in order to bootstrap the model using the hierarchical fitting procedure mritotal. The resulting linear transformation, TA, is flipped about the $\mathrm{X}$-axis and used as the initial transformation for registering the mirror image to the ICBM152 model yielding TF. TF is then flipped about the $\mathrm{X}$-axis in order to be averaged with TA. Afterwards, the averaged transformation is applied to the aligned data-set and the flipped averaged transformation to the mirror image. Then all the resulting data-sets were averaged as well as all transformations. The inverted average scaling factor is then applied to the averaged data-set which generates the next generation model. This is done in order to scale the model into the average size of all datasets used. Iteration 1 performs the same fitting procedure as iteration 0 to the model generated in iteration 0 .

A general overview of the slightly different non-linear fitting process which starts at iteration 2 is shown in Fig. 1. Here all subject data-sets (aligned and flipped) are non-linearly matched to the model generated in the previous iteration where the linear transformations created in iteration 1 are used as initial transformations. For each subject, the resulting transformations TA and TF are combined by flipping TF and averaging them ignoring the linear component. This averaged transformation is then concatenated with the linear transform (created in iteration 1) and the inverse of the scaling factor described with iteration 0 (Fig. 1. index I).

The composed transformation TX is then applied to aligned subject data and a flipped version of the transformation to the flipped data (see Fig. 1. index J). The resulting volumes are then averaged separately (Fig. 1. indexes K); aligned 


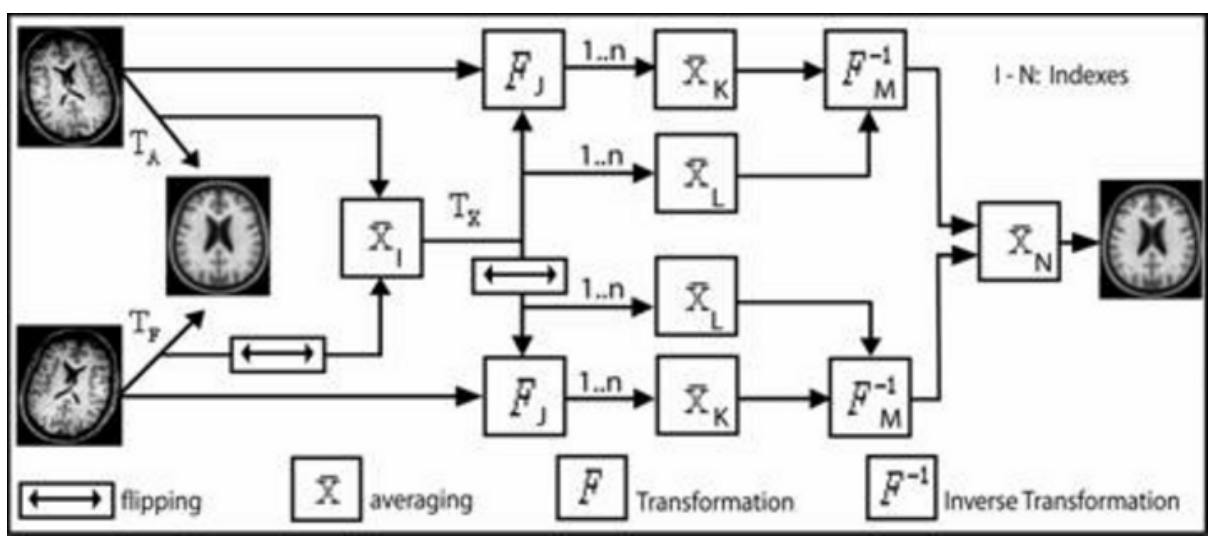

Fig. 1. Schmatic of non-linear fitting and averaging procedure

and flipped. In order to generate the next generation model the average of the aligned transformations (linear component ignored, Fig. 1. index L) must be inverse applied to the average aligned volume (Fig. 1. index M) and respectively to the flipped data. The results of these transformations are averaged (Fig. 1. in$\operatorname{dex} \mathrm{N}$ ) which represents the next gen-eration model. In this work, the non-linear fitting process was repeated nine times by constantly increasing the registration resolution. Table 1 shows detailed information regarding the registration process.

Table 1. Number of iterations used during each stage of non-linear fitting. Iterations 0 and 1 are linear, iterations 2 to 10 are non-linear with a deformation grid resolution from $16 \mathrm{~mm}$ to $1 \mathrm{~mm}$.

\begin{tabular}{lllllll}
\hline Iteration & Target Model & \multicolumn{1}{c}{$16-168 /[8,4] 4 /[4,2,2] 2 /[2,2] 1$} \\
\hline 0 & symmetric ICBM152 & - & - & - & - & - \\
1 & model generated in iteratoin 0 & - & - & - & - & - \\
2 & model generated in iteratoin 1 & 35 & - & - & - & - \\
3,4 & model generated in iteratoin 2,3 & 35 & 35 & - & - & - \\
$5,6,7$ & model generated in iteratoin $4,5,635$ & 35 & 25 & - & - \\
8,9 & model generated in iteratoin 7,8 & 35 & 35 & 25 & 10 & - \\
10 & model generated in iteratoin 9 & 35 & 35 & 25 & 10 & 5 \\
\hline
\end{tabular}

\subsection{Manual Hippocampus Segmentation}

The HC was manually traced in 118 of the patient data sets in both hemispheres using the method described by Pruessner et al [15. In brief the method consists of: aligning all images to ICBM space via a linear transformation to assist with orientation during tracing, isotropic resampling of all data to $1 \mathrm{~mm}$ and tracing the chosen structure via the use of simultaneous coronal, transverse and sagittal views. 


\subsection{Comparison; Manually Traced - Automatically Segmented Hippocampi}

As all patient data sets have now been non-linearly matched to the final average model-based automated segmentations of regions that exhibit sufficient image contrast such as the hippocampus are possible as per Collins et al [14]. To compare manually traced hippocampi against automatically segmented ones, the first half of 118 manually traced hippocampi is transformed on the non-linear average model (using the subject data to model transformations that were obtained in the last iteration of model generation) to create a $\mathrm{HC}$ on the target volume. This $\mathrm{HC}$ model is then back-transformed onto the individual patients anatomy of the second half. Kappa correlation is used as a measure of agreement between the manually traced hippocampus and the automatic segmented one for each subject. This process is repeated by transforming the second half of the manually traced hippocampi on the non-linear average and back-transforming the average to onto the individual subjects of the first half.

\section{Results}

Fig. 2 demonstrates the registration results of two aligned subjects during the progres-sion towards a symmetric average model. In this series of images the shift towards symmetry is very apparent in the later iterations. This effect is first exhibited in larger structures (e.g. ventricles) followed by more local structures (e.g. smaller sulci). The last iterations demonstrate the ability of the non-linear matching methodology to find a mean between different patients anatomy. The effect of anatomical normalization can easily be seen by comparing the anatomy of patient 1 and 2 (Fig. 2) in iterations 1 and 10. After non-linear registration the different anatomies (iteration 1) have become very similar (iteration 10).

The effect of normalization of anatomy can also be seen in the associated average images, here the most obvious effect during the iteration towards a symmetric model is the reduction in variance associated with increase in contrast as the model evolves. This effect can be seen in Fig. 3 as a reduction in standard deviation. It should also be noted that in later iterations the mean image contains more anatomical information as more and more structures are matched. This is also reflected in the standard deviation images. Automatic segmentation as described in section 2.5 was compared against expert manual delineation [15] of the hippocampus in 118 non-demented community volunteers.

The Kappa mean value (for the process where the average hippocampus created from the second half of the data-set cohort was back-transformed to the individual patient data-sets of the first half) was 0.770 accompanied by a standard deviation of 0.027 . For the process which was done in the opposite way, the Kappa mean was 0.789 with a standard deviation of 0.032 . However, it must be noted that left/right comparisons in the proposed methodology will be without bias. For this subject cohort, left/right comparison of the hippocampus volume shows no significant difference. Fig. 4 (a) shows the non-liner model in which 

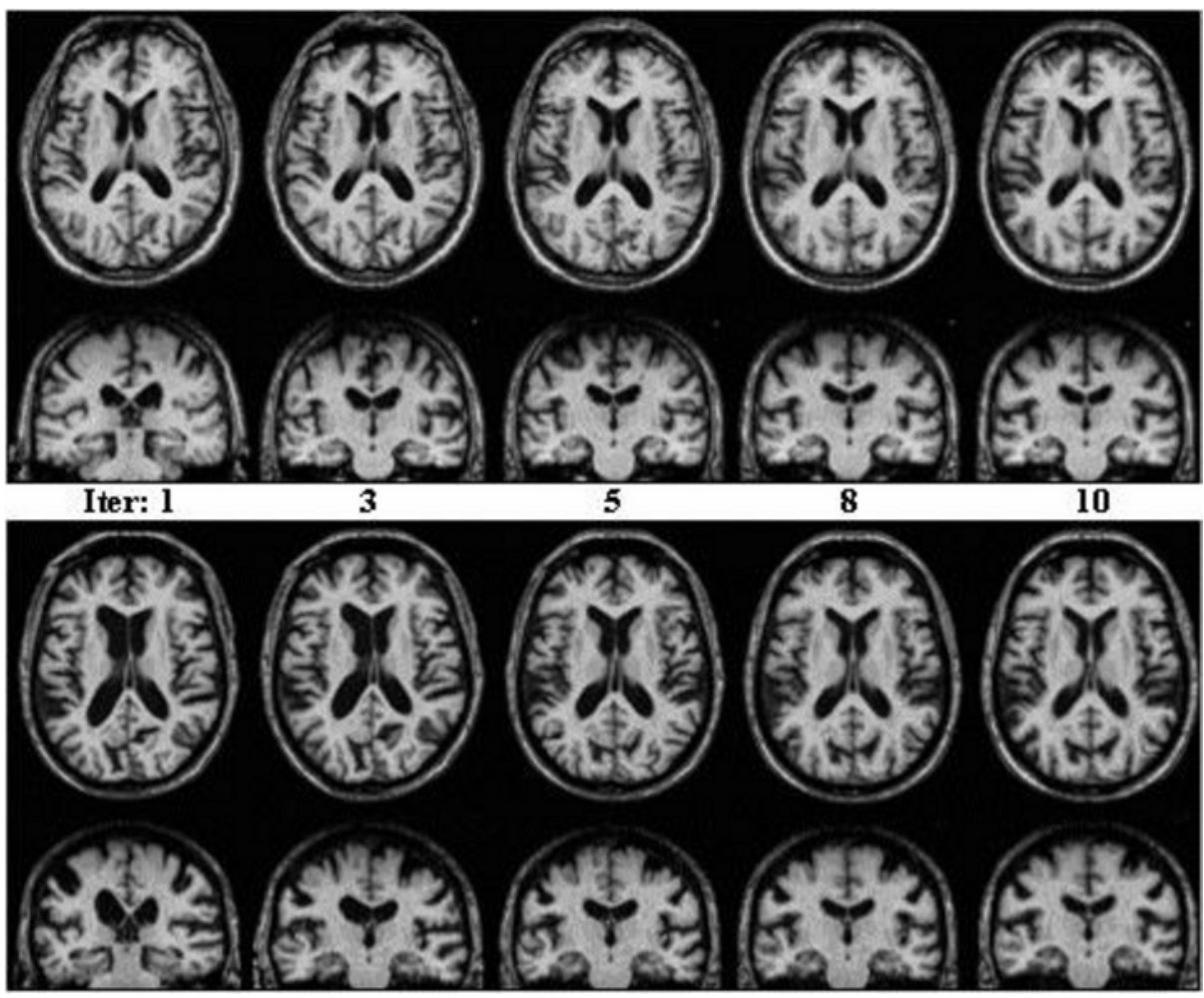

Fig. 2. Registration results for two patients during a stepwise matching to the evolving model of average structure

the averaged hippocampus is outlined, a randomly selected example for manually tracing is shown in (b) and (c) outlines the correspond-ing automatically segmented hippocampus.

\section{Discussion}

This paper has presented a method whereby automated model based segmentation of symmetric structures is made possible. This is done by registering complementary original and flipped images to a target simultaneously, this feature is important to ensure bias is not introduced at any stage of the model generation. It is also important to note that during iterations registration begins with each of the images in their initial space. This constraint ensures that no bias is introduced towards a particular interme-diate model during the construction of the final model.

It should be noted that this work embodies a small extension to the work done by Guimond on iterative atlasing, there are however two critical differences. The first is that after each interation of model building, the inverse of the average 


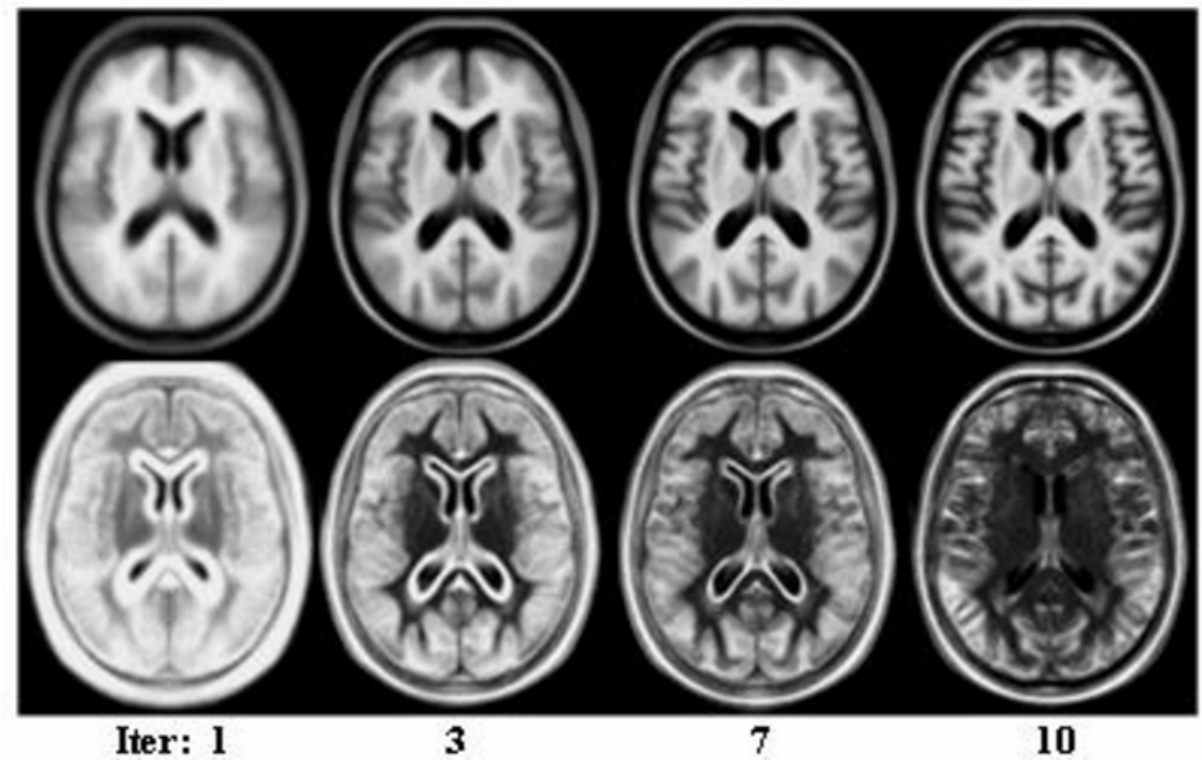

Fig. 3. Successive, reduction in variance and increase in contrast as the model evolves. Top row: Mean images of 153 averages (aligned data-sets), bottom row: Standard deviation images (aligned data-sets)

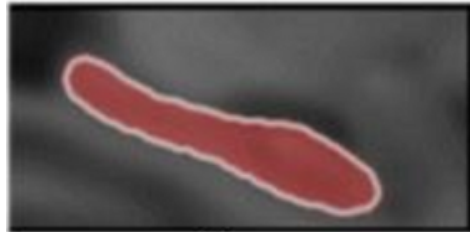

(a)

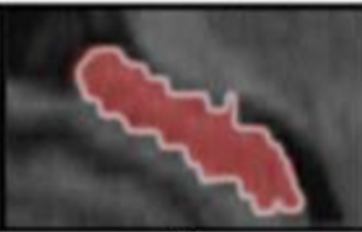

(b)

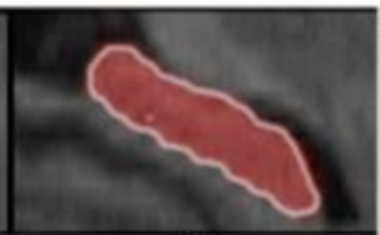

(c)

Fig. 4. Comparison of manually tracing and automatic segmentation; (a) non-liner model, (b) manually tracing - and (c) automatic segmentation of the hippocampus

transformation is applied to the model, this controls for possible bias in the chosen method of registration. The second is that in this case we are building a symmetric model. Whilst a comparison of the two methods for segmenting substructures would seem a logical test for the new method, the results will be of little utility if only because only the results obtained using the new method will be unbiased towards the left or right sub-structure thas has been chosen. That said, givan that both approaches use the same underlying registration technique, the reults will be very similar.

During the generation of the model it was found that a hierarchical approach to fitting as detailed in Table 1 is critical. As such only structures that exhibit high anatomic consistency (eg motor strip, ventricles) across a population are 
evident in the eventual model. Due to the nature and extent of processing required to build such a model the computing time taken to generate this model is large. Approximately 2 weeks of dedicated processing time on a cluster of 16 Linux machines. Whilst this may seem an inordinate amount of processing time, it must be noted that once a model is built, the time taken to then match a novel subjects anatomy to the model is on the order of minutes.

For the chosen registration techinque (ANIMAL) it was found that the numbers of iterations given in Table 1 were optimal for our dataset and thus likely for most elderly T1 data. Clearly this number of iterations will need to be tuned for data that is not of this type. In our case, an iterations fitting was continued untill the Standard Deviation image stabilised for that particular stage. Once this was achieved only then was fitting allowed to progress a finer scale.

The utility of a method whereby features can be automatically identified and ex-tracted from cortical data is far reaching but can be problematic [16]. One of these problems is the issue of symmetric bias when comparing structures leftright. The methodologies presented in this paper are intended to enhance the existing methods whereby models are built using non-linear registration with no corresponding impact on accuracy of automated segmentation. The most obvious benefit is the ability to perform comparisons of structures that are by nature asymmetric but posses enough symmetry to allow left-right comparisons to be made.

\section{References}

1. Guimond A, Meunier J, Thirion J-P.: Average brain models: A Convergence Study Computer Vision and Image Understanding. 1999 77(2):192-210

2. Filippi M, Horsfield MA, Bressi S, Martinelli V, Baratti C, Reganati P, Campi A, Miller DH, Comi G.: Intra- and inter-observer agreement of brain MRI lesion volume measure-ments in multiple sclerosis. A comparison of techniques. Brain. 1995;118(6):1593-600

3. Toga AW, Samaie M, Payne BA.: Digital rat brain: a computerized atlas. Brain Res Bull 1989 Feb;22(2):323-33

4. Dann R, Hoford J, Kovacic S, Reivich M, Bajcsy R.: Evaluation of elastic matching system for anatomic (CT, MR) and functional (PET) cerebral images. J Comput Assist Tomogr 1989 Jul-Aug;13(4):603-11

5. Greitz T, Bohm C, Holte S, Eriksson L.: A computerized brain atlas: construction, anatomi-cal content, and some applications. J Comput Assist Tomogr 1991 JanFeb;15(1):26-38.

6. Toh MY, Falk RB, Main JS.: Interactive brain atlas with the Visible Human Project data: development methods and techniques. Radiographics 1996 Sep;16(5):1201-6

7. Toga AW, Thompson PM.: Maps of the brain. Anat Rec 2001 Apr15;265(2):37-53

8. Gee JC, Reivich M, Bajcsy R.: Elastically deforming 3D atlas to match anatomical brain images. J Comput Assist Tomogr 1993 Mar-Apr;17(2):225-36

9. Thompson PM, Toga AW.: A Surface-Based Technique for Warping 3-Dimensional Images of the Brain. IEEE Trans on Med Imag. 1996;15(4):1-16

10. Davatzikos C.: Spatial normalization of 3D brain images using deformable models. J Comput Assist Tomogr 1996 Jul-Aug;20(4):656-65 
11. Wang L, Joshi SC, Miller MI, Csernansky JG.: Statistical analysis of hippocampal asymme-try in schizophrenia. Neuroimage. 2001 Sep;14(3):531-45

12. Evans AC, Collins DL, Milner B.: An MRI-based stereotaxic atlas from 250 young normal subjects. Society Neuroscience Abstracts. 1992;18:408

13. Sled JP, Zijdenbos AP and Evans AC.: A non-parametric method for automatic correction of intensity non-uniformity in MRI data. IEEE Transactions on Medical Imaging. 1998;17(1):87-97

14. Collins DL, Holmes C, Peters T, Evans A.: Automatic 3D Model-Based Neuroanatomical Segmentation. Human Brain Mapping. 1995;3:190-208

15. Pruessner JC, Li LM, Serles W, Pruessner M, Collins DL, Kabani N, Lupien S, Evans AC.: Volumetry of Hippocampus and Amygdala with High-Resolution MRI and Three-dimensional Analysis Software: Minimizing the Discrepancies between Laboratories. Cerebral Cortex. 2000;10(4):433-442

16. Thompson PM, Woods RP, Mega MS, Toga AW.: Mathematical/computational challenges in creating deformable and probabilistic atlases of the human brain. Human Brain Mapping 2000 Feb;9(2):81-92

17. B. M. Dawant, A. P. Zijdenbos, and R. A. Margolin.: "Correction of Intensity Variations in MR Images for Computer-Aided Tissue Classification", IEEE Trans. Med Imaging, Vol. 12, 1993. 